\title{
Development of Microfluidic Device Coupled with Post-wall Waveguide for Microwave Heating at $24.125 \mathrm{GHz}$
}

\author{
Kaito Fujitani, ${ }^{1 *}$ Mitsuyoshi Kishihara, ${ }^{2}$ Tomoyuki Nakano, ${ }^{1}$ \\ Ryota Tanaka, ${ }^{1}$ Akinobu Yamaguchi, ${ }^{1}$ and Yuichi Utsumi ${ }^{1}$ \\ ${ }^{1}$ Laboratory of Advanced Science and Technology for Industry, University of Hyogo, \\ 3-1-2 Kouto, Kamigori, Ako, Hyogo 678-1205, Japan \\ ${ }^{2}$ Okayama Prefectural University, 111 Kuboki, Soja, Okayama 719-1197, Japan
}

(Received January 29, 2021; accepted March 30, 2021; online published April 14, 2021)

Keywords: lab-on-a-chip, microwave, post-wall waveguide, fluorinated ethylene propylene, indium tin oxide

We have developed a micro-chemical chip coupled with a post-wall waveguide for various chemical reactions monitored in situ during microwave irradiation at $24.125 \mathrm{GHz}$. This device has a structure in which a microchannel passes between metal post-walls. Fluorinated ethylene propylene (FEP) resin is used for the microchannel's structure, and glass with an indium tin oxide (ITO) film is also used to prevent the leakage of microwaves from the top and bottom of the microchannel. FEP is a feasible material for use as a waveguide and a reactor structural material for chemical synthesis owing to its low dielectric loss, high heat resistance (up to 200 ${ }^{\circ} \mathrm{C}$ ), and chemical inertness. In addition, its excellent transparency in the visible region also allows the optical monitoring of various reactions during microwave heating. An ITO film was sputtered on the glass, and the resulting glass had a microwave transmittance of $-27.5 \mathrm{~dB}$. When water and ethylene glycol were heated with a microwave input power of $4.0 \mathrm{~W}$, the temperature rose to 82.7 and $91.5{ }^{\circ} \mathrm{C}$, respectively. In addition, by observing the inside of the microchannel during microwave irradiation, it was possible to confirm the state of boiling induced in the vicinity of the microwave input section. This is consistent with the temperature distribution in the simulation results. This device enables the rapid heating of solvents by microwave irradiation and optical monitoring of the microwave-induced chemical reactions.

\section{Introduction}

In recent years, research and development on microwave chemistry using microwaves as a heat source has markedly advanced. The high reaction yield, selectivity, and rapidity, which are characteristics of microwave heating, have been demonstrated and applied in various fields such as organic/inorganic chemistry, polymer chemistry, and metal chemistry, and microwave heating is recognized as an innovative chemical synthesis method. ${ }^{(1-5)}$

Currently, rectangular waveguide systems and multimode cavities are often used as the basic structure of microwave chemistry equipment with high-power sources of up to several $\mathrm{kW}$ in the

*Corresponding author: e-mail: kit1129@lasti.u-hyogo.ac.jp

https://doi.org/10.18494/SAM.2021.3308 
2.45 GHz band. ${ }^{(6,7)}$ However, integrated sequential chemical unit operations in microwave cavities using a flow system and their monitoring, which are indispensable for performing multiple/parallel synthesis at a high speed and high yield, have not yet been realized because it is necessary to isolate the reaction field in the metallic microwave cavities in conventional systems. As one of the promising approaches to resolve the above issues for chemical synthesis, we have proposed a lab-on-a-chip (LOC) in which microchannels are coupled to a post-wall waveguide using microwaves of the $24 \mathrm{GHz}$ band. ${ }^{(8,9)}$ The LOC contains a microchannel and can be freely combined with complex unit chemical operations with small amounts of source reagents and products. It also has a fast reaction time and energy conservation, so its application to biomedical and chemical syntheses has been actively attempted. ${ }^{(10,11)}$ The post-wall waveguide, whose size matches the microfluidic channel in the LOC, can effectively minimize the microwave leakage by arranging metal columns at regular intervals. ${ }^{(12,13)}$ By using microwaves at $24.125 \mathrm{GHz}$, the microwave absorption of water is higher than that of commercial microwaves at $2.45 \mathrm{GHz}$, so it is possible to heat water even at a lower input. However, when using the proposed chip, it was difficult to observe chemical reactions directly from outside the chip through the structural material due to the inhibition by the metal shield plates and the low optical transparency of polytetrafluoroethylene (PTFE) when observing the reaction using these device waveguide materials.

To solve the problem, we have improved a micro-chemical chip with a post-wall waveguide using indium tin oxide (ITO) and fluorinated ethylene propylene (FEP) as the structural materials. ITO is a transparent conductive film and is applied in various fields such as liquid crystal displays and solar cells. It also has excellent light transmission in the visible light region. Similarly, FEP has excellent light transmittance, high chemical stability, and low dielectric loss. In this study, we prototyped a chip-size device capable of microwave irradiation at $24.125 \mathrm{GHz}$ and evaluated its performance by simulating the temperature rise of liquids and optical temperature measurement from outside the chip using fiber optics.

\section{Materials and Methods}

\subsection{Design of microchip with microchannel combined with post-wall waveguide}

The microchip consists of metallic post-walls and a microchannel passing between them. Figure 1(a) shows a schematic diagram of continuous microwave irradiation using the post-wall waveguide and a simple microchannel. The relative permittivity $\varepsilon_{r}$ of the materials that make up the microchannel is 2.1. Metallic posts of radius $r=0.365 \mathrm{~mm}$ are arranged at intervals of $a_{f} / 4{ }^{\prime}=$ $1.7 \mathrm{~mm})$. The length of the connector $\left(l_{d}\right)$ and the microchannel width $g_{w}$ are 3.2 and $1.6 \mathrm{~mm}$, respectively. In order to connect the microchannel to the inside of the waveguide, the distance between the post-walls on both sides, through which the microchannel passes, was adjusted to $1.4 \mathrm{~mm}$. Figure 1(b) shows a cross-sectional diagram of the microchip composed of the post-wall waveguide and the microchannel. The length of the central conductor of the coaxial line $\left(l_{c}\right)$ is $1.5 \mathrm{~mm}$, and the height $\varepsilon_{0}$ of the air layer that is machined above it is $0.5 \mathrm{~mm}$. The post-wall waveguide was formed by stacking 1.5 -mm-thick FEP sheets. The height of the microchannel $\left(g_{h}\right)$ is $1.6 \mathrm{~mm}$, so the cross section of the microchannel is a square structure with a side of 
(a)

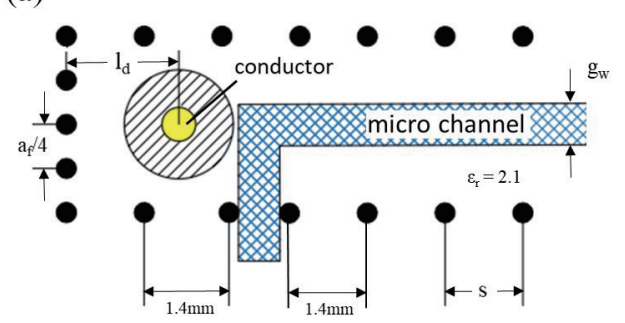

(b)

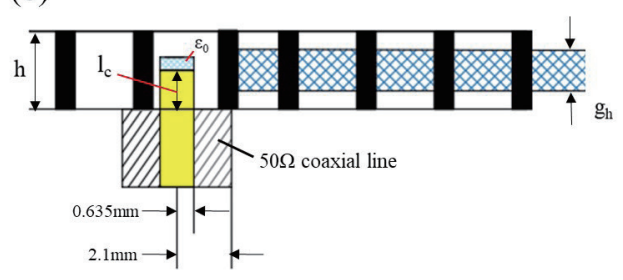

Fig. 1. (Color online) (a) Schematic diagram of the basic structure using the post-wall waveguide with a straight section. (b) Cross-sectional diagram of the basic structure using the post-wall waveguide.

$1.6 \mathrm{~mm}$. The height $h$ of the waveguide is $3 \mathrm{~mm}$, the width $a_{f}$ of the waveguide is $6.8 \mathrm{~mm}$, and the distance $s$ between the post-walls is $1.8 \mathrm{~mm}$. In this design, the dimensions are adjusted so that the cutoff frequency is around $16 \mathrm{GHz}$ and the attenuation is about $0.05 \mathrm{~Np} / \mathrm{m}$ or less. Generally, the height of the post-wall waveguide is less than half of the wavelength, and it has a constant cross section in the height direction, so the propagation mode is TE10-like, which is similar to the TE10 mode of a conventional metallic waveguide.

Figure 2(a) shows a perspective diagram of the prepared microchip. This microchannel has a curved shape, and the liquid can be smoothly manipulated from the outside. In addition, two post-walls are added near the microchannel to prevent the leakage of microwaves, whose wavelength decreases in the liquid. The distance between the additional metal columns $a_{f} / 4$ is $1.7 \mathrm{~mm}$. This microchip forms the microchannel and post-wall waveguide with two FEP sheets (thickness: $1500 \mu \mathrm{m}$ ), and is sandwiched between two pieces of glass, on which an ITO film of $3 \mathrm{~mm}$ thickness was formed [Fig. 2(b)]. By using the post-wall waveguide, it is possible to easily incorporate the microchannel by utilizing the space between the metal columns while keeping the microwave energy confined in the waveguide. In addition, it can be freely designed for complex chemical unit operations using multiple microchannels. The microfluidic device can combine the following functions.

(1) Injection of solution into the microchannel.

(2) Microwave irradiation into the post-wall waveguide.

(3) Removal of reacted solution after microwave irradiation.

Furthermore, it enables reactions to be monitored during microwave irradiation because of the use of the ITO film on the glass and the FEP. FEP is used in this microchip because it has excellent electrical properties (low dielectric loss), thermal properties (robust up to $200{ }^{\circ} \mathrm{C}$ ), and chemical properties. Polydimethylsiloxane (PDMS), cycloolefin polymer (COP), and poly- 


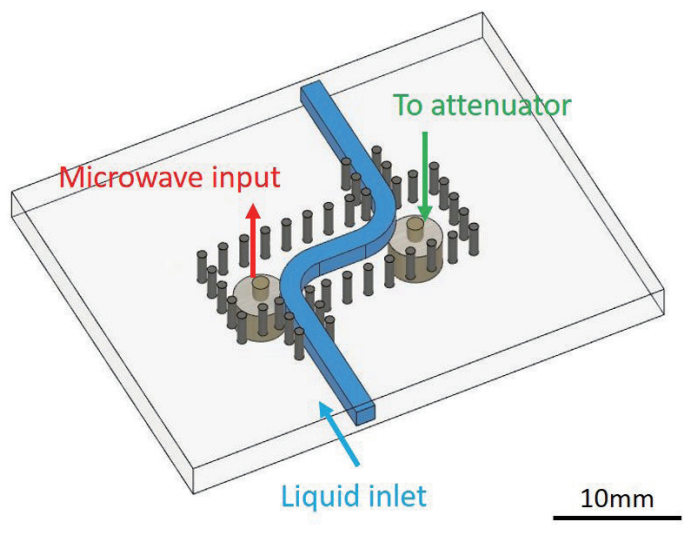

(a)

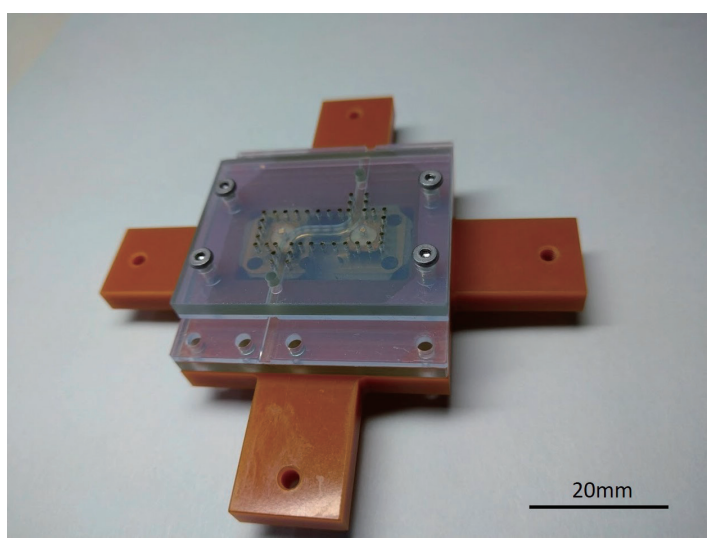

(b)

Fig. 2. (Color online) (a) Perspective diagram of designed structure of microchannel embedded in the post-wall waveguide. (b) Photograph of the fabricated microchip.

methyl-methacrylate resin (PMMA) ${ }^{(14,15)}$ are generally used as materials for microfluidic devices. FEP is suitable for organic synthesis and drug synthesis by microwave irradiation because it is advantageous over these materials in terms of the above properties.

\subsection{Experiment}

In order to confirm the validity of the design and the numerical simulation results, we measured the temperature rises of water and ethylene glycol and monitored their reaction during microwave irradiation. As shown in Fig. 2(b), the microchip was fabricated by forming a machining flow path pattern on two FEP sheets and superimposing them to form a microchannel. After that, a metal column with $r$ of $0.365 \mathrm{~mm}$ was inserted to create the post-wall waveguide arrays [Fig. 3(a)], where Ni-coated Fe was used as the structural material. Two holes were also made in the glass to attach the connector, and metal walls were formed with gold to prevent microwaves from leaking [Fig. 3(b)]. Then, a thin ITO film was deposited on the glass by RF sputtering. ITO $\left(90 \mathrm{wt} \% \mathrm{In}_{2} \mathrm{O}_{3} 10 \mathrm{wt} \% \mathrm{SnO}_{2}\right.$ ) was used as the target, and sputtering was performed at a pressure of $0.6 \mathrm{~Pa}$ in an Ar atmosphere. The RF output was $200 \mathrm{~W}$ and the sputtering was performed for $3 \mathrm{~h}$ while rotating the table on which the sample was placed. The film thickness was measured to be $760 \mathrm{~nm}$ using a Dektak IIa profilometer (VEECO/SLOAN). Figure 4(a) shows the microwave transmittance of the ITO film sputtered on the 1-mm-thick glass. According to Fig. 4(a), the transmittance of the microwave with a frequency of $24.125 \mathrm{GHz}$ was about $-27.5 \mathrm{~dB}$. Figure 4(b) shows the transmittance of the ITO glass in the visible light region. It shows a transmittance of approximately 70 to $80 \%$ in the visible light region of 450 to $700 \mathrm{~nm}$. Generally, the transmittance of an ITO film formed by sputtering from the ultraviolet region to the infrared region depends on the oxygen partial pressure in the sputtering atmosphere, the heating temperature of the substrate, and the heating temperature of post-annealing. ${ }^{(16-18)}$ Finally, an SMA connector was attached for the input and output of microwaves. The temperature rise of the solvent upon microwave heating was measured using an optical fiber cable (FS100-2M Anritsu-Meter Co., Ltd.). The microwave output was $4 \mathrm{~W}$. 


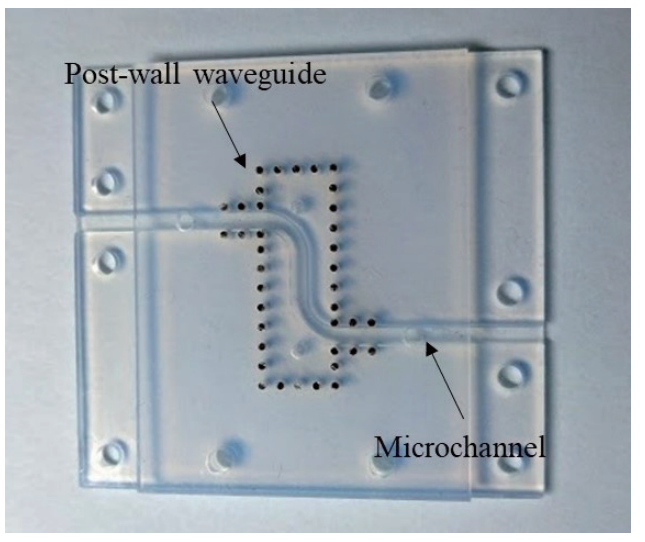

(a)

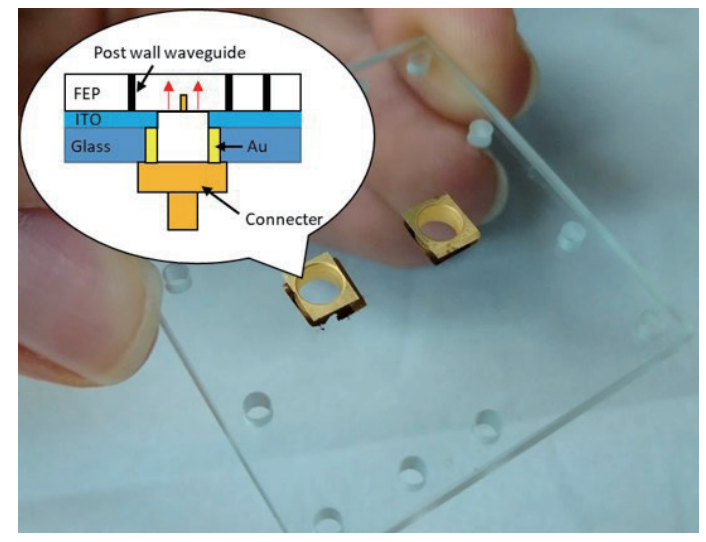

(b)

Fig. 3. (Color online) (a) Microchannel of FEP with post-wall waveguide. (b) Metallic wall of glass hole.

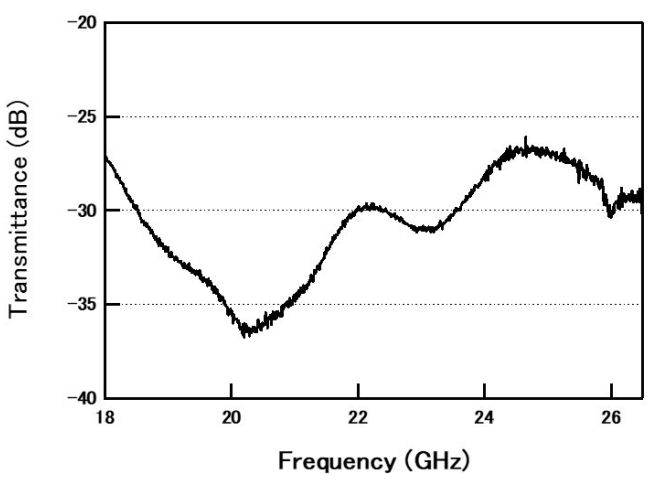

(a)

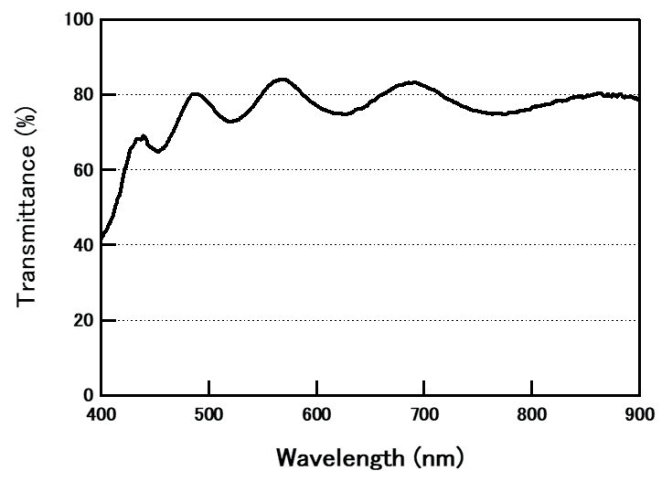

(b)

Fig. 4. (a) Microwave transmittance of the ITO film sputtered on the 1-mm-thick glass at frequencies around 24.125 GHz. (b) Transmittance of the ITO film sputtered on the 1-mm-thick glass in the visible light region.

Figure 5 shows the experimental system. The microwave emitted from the $24.125 \mathrm{GHz}$ transmitter is amplified by a $4 \mathrm{~W}$ power amplifier and emitted to the microchip with an output of $4 \mathrm{~W}$. Also, the microwave reflected to the amplifier is blocked by an isolator installed between the amplifier output and the post-wall waveguide. It is necessary for the terminator to consume the microwave power that was not absorbed by the solvent, because this microchip has a nonresonator waveguide structure. Therefore, the output side uses a $50 \Omega$ terminator (maximum $10 \mathrm{~W})$ to attenuate the microwave.

\section{Results and Discussion}

Figure 6 shows the temperature distribution of water after $180 \mathrm{~s}$ of microwave irradiation simulated by COMSOL Multiphysics. As the parameters of the pure water in this simulation, we assumed a thermal conductivity of $0.615 \mathrm{~W} /(\mathrm{mK})$, a heat capacity of $4.2 \mathrm{~kJ} /(\mathrm{kgK})$, and a permittivity of 32.5-j34.98. The microwave input power to the coaxial port was $1 \mathrm{~W}$ and the frequency was $24.125 \mathrm{GHz}$. The room temperature was $24{ }^{\circ} \mathrm{C}$. This simulation indicated that the 


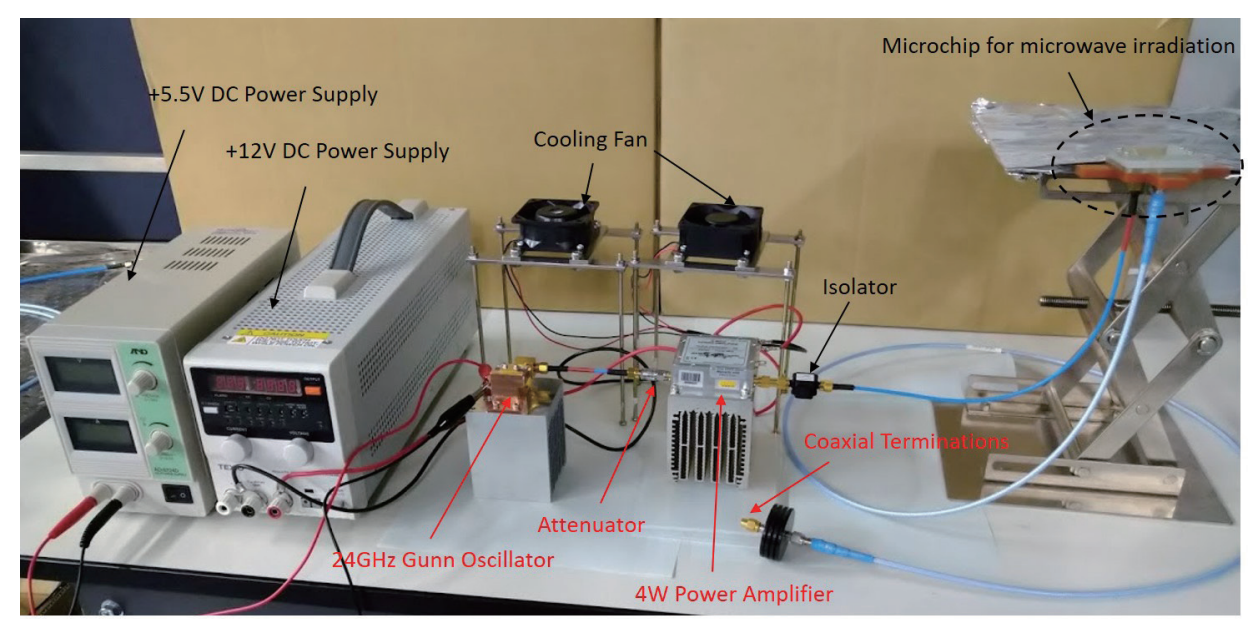

Fig. 5. (Color online) Photograph of experimental setup.
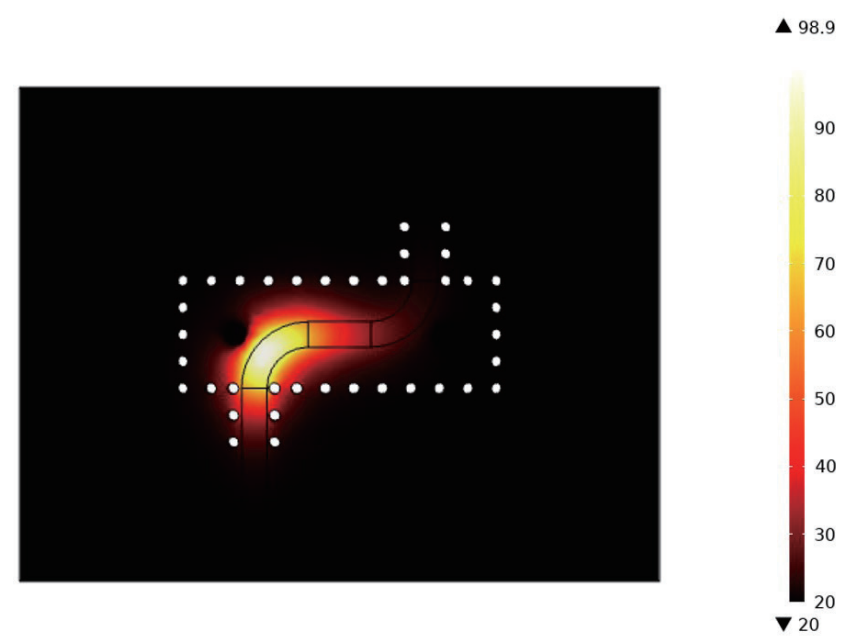

Fig. 6. (Color online) Theoretical temperature distribution of water after $180 \mathrm{~s}$ of microwave irradiation simulated using COMSOL Multiphysics.

maximum temperature increased to $97.9^{\circ} \mathrm{C}$ near the coaxial port. In addition, the temperature decreased as the distance from the coaxial port that outputs microwaves increased. This indicates that the thermal energy locally heats the inside of the microchannel. Figure 7(a) shows the simulation results when the microchannel in the post-wall waveguide was irradiated with microwaves with input powers of 1 and $4 \mathrm{~W}$. In the case of $4 \mathrm{~W}$ input power, the temperature increased to $330{ }^{\circ} \mathrm{C}$, where thermal diffusion to the outer area of the waveguide and the phase transition from liquid to gas were not considered. Figure $7(\mathrm{~b})$ shows a graph of the water temperature during microwave irradiation with $4 \mathrm{~W}$ input power when $100 \mu \mathrm{L}$ of water was injected into the microchannel and the temperature was measured by inserting an optical fiber thermometer from the microchannel on the microwave input section. In this experiment, the water temperature increased from 22.5 to $82.7^{\circ} \mathrm{C}$, whereas the maximum temperature for $4 \mathrm{~W}$ irradiation reached $330^{\circ} \mathrm{C}$ in the simulation, showing a large difference. The factors causing the 


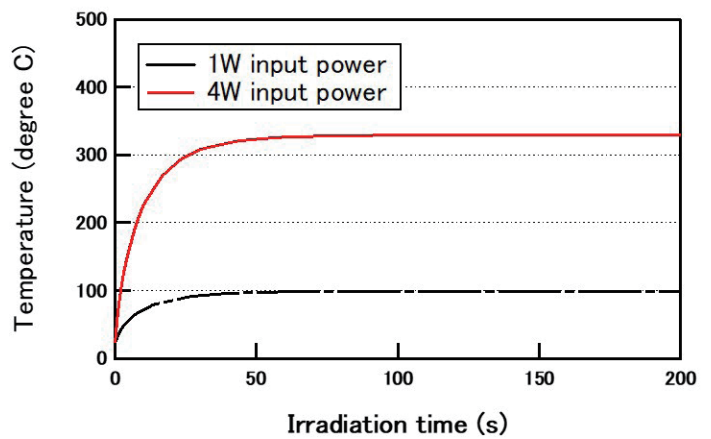

(a)

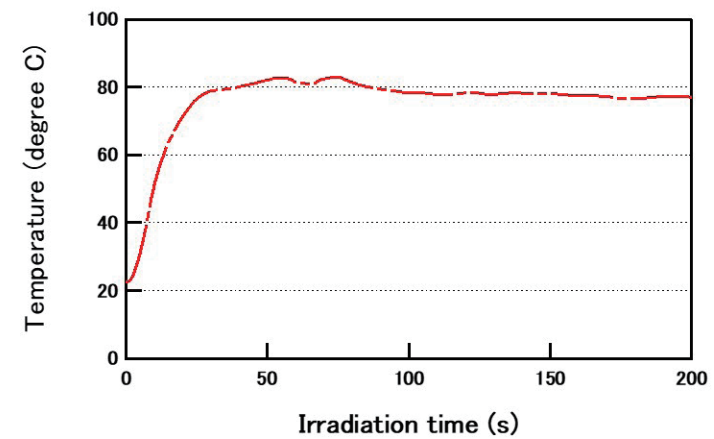

(b)

Fig. 7. (Color online) (a) Water temperature during microwave irradiation with input powers of 1 and $4 \mathrm{~W}$ simulated using COMSOL Multiphysics. (b) Actual water temperature during microwave irradiation at $4 \mathrm{~W}$ input power.

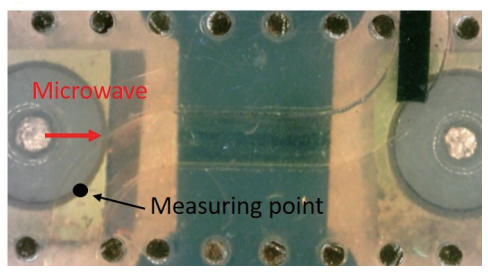

(a)

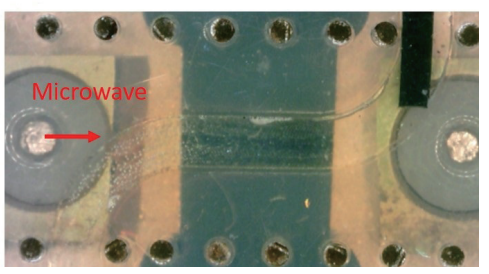

(b)

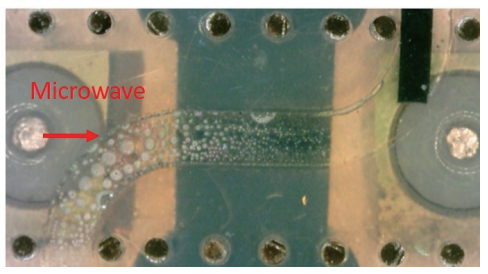

(c)

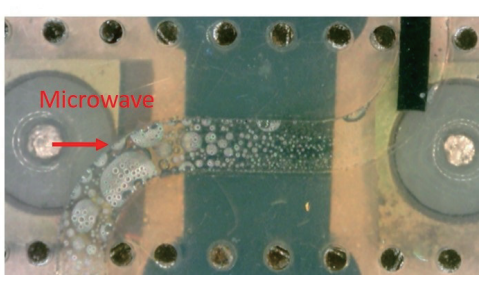

(d)

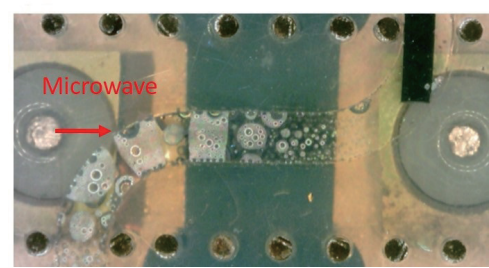

(e)

Fig. 8. (Color online) Photographs of ethylene glycol irradiated with microwaves for (a) $0 \mathrm{~s}$, (b) $10 \mathrm{~s}$, (c) $20 \mathrm{~s}$, (d) $30 \mathrm{~s}$, and (e) $60 \mathrm{~s}$.

lower maximum temperature in the experiment are expected to be the heat of vaporization due to the phase transition and the heat diffusion to the external support structure, which were not considered in the simulation. Figure 8 shows photographs taken when water was irradiated with microwaves at $24.125 \mathrm{GHz}$. After $10 \mathrm{~s}$ of microwave irradiation [Fig. 8(b)], bubble generation was first observed, which started from the microwave input section and finally occurred near the output section. This indicates that the temperature was highest in the microwave input section, as shown in Fig. 6, which is qualitatively consistent with the temperature distribution of the simulation results. In addition, the effects of the machining accuracy and leakage from gaps in the FEP and ITO film glass are also considered have made a difference in the calculation results. It is expected that the experimental value would have been closer to the simulation result if the FEP and ITO film glass had been adhered to each other to improve the processing accuracy and fill the gap between the FEP and ITO glass. 
A similar experiment was also performed using ethylene glycol (boiling point: $197{ }^{\circ} \mathrm{C}$ ), which has a boiling point higher than that of water. Figure 9 shows the temperature change of ethylene glycol when irradiated with microwaves at $4 \mathrm{~W}$ input power. The temperature rose to $75.3{ }^{\circ} \mathrm{C}$ in $60 \mathrm{~s}$, and continued to rise thereafter up to a maximum value of $91.5{ }^{\circ} \mathrm{C}$. The measuring point was the same as that when water was heated. In addition, in the reaction of ethylene glycol shown in Fig. 10, a small quantity of bubbles originated from along the microchannel wall. This indicates that ethylene glycol can be sufficiently heated with a microwave input power of $4 \mathrm{~W}$ or more. Ethylene glycol solution is used as a solvent for complex and organic syntheses using microwaves, and it is expected that organic syntheses under microwave irradiation can be performed using this device structure while monitoring the reaction.

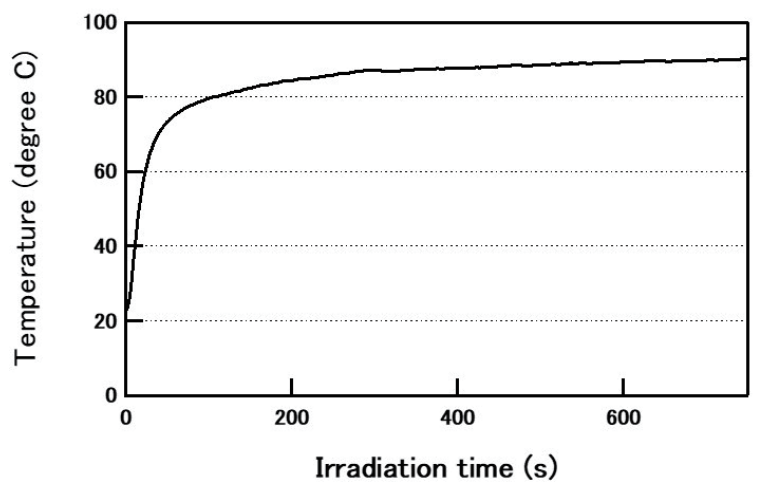

Fig. 9. (Color online) Ethylene glycol temperature during microwave irradiation at $4 \mathrm{~W}$ input power.

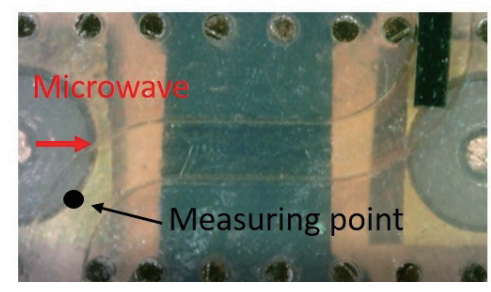

(a)

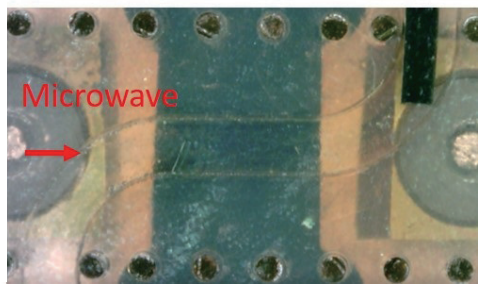

(b)

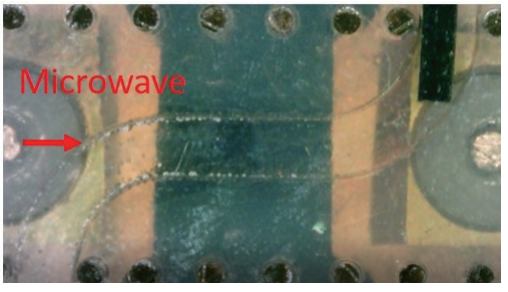

(c)

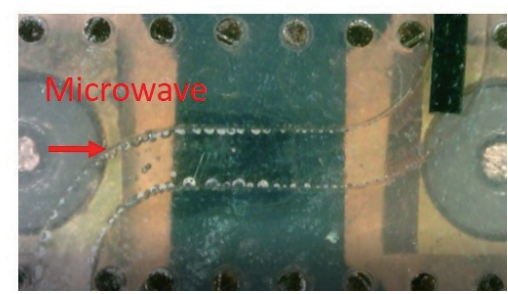

(d)

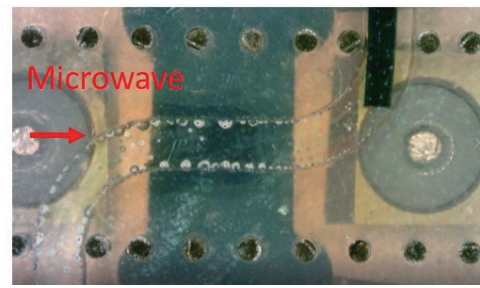

(e)

Fig. 10. (Color online) Photographs of ethylene glycol irradiated with microwaves for (a) $0 \mathrm{~s}$, (b) $30 \mathrm{~s}$, (c) $90 \mathrm{~s}$, (d) $180 \mathrm{~s}$, and (e) $300 \mathrm{~s}$. 


\section{Summary and Conclusions}

In this study, we fabricated a microchip for monitoring reactions during microwave irradiation. FEP, which has chemical stability and heat resistance up to $200{ }^{\circ} \mathrm{C}$, was used as the structural material for the microchannel. ITO films were also used as the waveguide walls at the top and bottom of the microchannel part to prevent microwave leakage. This made it possible to monitor the reaction in the visible light region while confining the microwaves to the waveguide. In addition, the microchannel was coupled into the waveguide through the gap in the post-wall. This device was evaluated by numerical simulation and temperature measurement while monitoring the reactions of water and ethylene glycol. The temperatures of water and ethylene glycol rose to 82.7 and $91.5{ }^{\circ} \mathrm{C}$, respectively, under microwave irradiation with a microwave input power of $4 \mathrm{~W}$. As a result of monitoring during microwave irradiation, it was confirmed that the boiling of the liquids was concentrated near the microwave input section. This is consistent with the temperature distribution in the simulation results. These results show that the proposed device for microwave heating is effective for reactions using water or ethylene glycol as a solvent. This device can contribute to the development of microfluidic devices with high functionality and high integration. In addition, it is expected to be applied to various fields that use microwave heating, such as the chemical, pharmaceutical, and industrial fields, because it is possible to monitor the reaction in the visible light region.

\section{Acknowledgments}

This work was supported by JSPS KAKENHI grant \#19K05142. We would like to thank Mr. Masao Noumi (DAIKIN INDUSTRIES) for providing the samples for this study.

\section{References}

1 C. O. Kappe: Angew. Chem. Int. Ed. 43 (2004) 6250. https://doi.org/10.1002/anie.200400655

2 F. Wiesbrock, R. Hoogenboom, and U. S. Schubert: Macromol. Rapid Commun. 25 (2004) 1739. https://doi. org/10.1002/marc.200400313

3 R. Roy, D. Agrawal, J. Cheng, and S. Gedevanishvili: Nature 399 (1999) 668. https://doi.org/10.1038/21390

4 C. Kappe and D. Dallinger: Nat. Rev. Drug Discovery 5 (2006) 51. https://doi.org/10.1038/nrd1926

5 S. A. Galema: Chem. Soc. Rev. 26 (1997) 233. https://doi.org/10.1039/CS9972600233

6 H. Yin, T, Yamamoto, Y. Wada, and S. Yanagida: Mater. Chem. Phys. 83 (2004) 66. https://doi.org/10.1016/j. matchemphys.2003.09.006

7 F. Mavandadi and A. Pilotti: Drug Discovery Today 11 (2006) 165. https://doi.org/10.1016/S13596446(05)03695-0

8 Y. Utsumi, A. Yamaguchi, T. Matsumura-Inoue, and M. Kishihara: Sens. Actuators, B 242 (2017) 384. https:// doi.org/10.1016/j.snb.2016.10.127

9 M. Kishihara, M. Isobe, K. Okubo, M. Takeuchi, A. Yamaguchi, and Y. Utsumi: 2020 Int. Symp. RadioFrequency Integration Technology Conf. (IEEE, 2020) 133-135. https://doi.org/10.1109/ RFIT49453.2020.9226210

10 Á. Ríos, M. Zougagh, and M. Avila: Anal. Chim. Acta 740 (2012) 1. https://doi.org/10.1016/j.aca.2012.06.024

11 P. S. Dittrich and A. Manz: Nat. Rev. Drug Discovery 5 (2006) 210. https://doi.org/10.1038/nrd1985

12 J. Hirokawa and M. Ando: IEEE Trans. Antennas Propag. 48 (2000) 1742. https://doi.org/10.1109/8.900232

13 J. Hirokawa and M. Ando: IEEE Trans. Antennas Propag. 46 (1998) 625. https://doi.org/10.1109/8.668903

14 J. C. McDonald and G. M. Whitesides: Acc. Chem. Res. 35 (2002) 491. https://doi.org/10.1021/ar010110q 
15 L. Yi, W. Xiaodong, and Y. Fan: J. Mater. Process. Technol. 208 (2008) 63. https://doi.org/10.1016/j. jmatprotec.2007.12.146

16 G. Goncalves, E. Elangovan, P. Barquinha, L. Pereira, R. Martins, and E. Fortunato: Thin Solid Films 515 (2007) 8562. https://doi.org/10.1016/j.tsf.2007.03.126

17 A. Chen, K. Zhu, H. Zhong, Q. Shao, and G. Ge: Sol. Energy Mater. Sol. Cells 120 (2014) 157. https://doi. org/10.1016/j.solmat.2013.08.036

18 E. Terzini, P. Thilakan, and C. Minarini: Mater. Sci. Eng., B 77 (2000)110. https://doi.org/10.1016/S0921$\underline{5107(00) 00477-3}$ 